\title{
A new model with solitary waves: solution, stability and quasinormal modes
}

\author{
Surajit Basak ${ }^{\dagger}$, Poulami Dutta Roy ${ }^{\#}$ and Sayan Kar \#* \\ $\dagger$ Condensed Matter Physics, Institute of Nuclear Physics, Polish Academy of Sciences, \\ ul. W. E. Radzikowskiego 152, PL-31342 Kraków, Poland and \\ \# Department of Physics, Indian Institute of Technology Kharagpur, 721 302, India
}

\begin{abstract}
We construct solitary wave solutions in a $1+1$ dimensional massless scalar $(\phi)$ field theory with a specially chosen potential $V(\phi)$. The equation governing perturbations about this solitary wave has an effective potential which is a simple harmonic well over a region, and a constant beyond. This feature allows us to ensure the stability of the solitary wave through the existence of bound states in the well, which can be found by semi-analytical methods. A further check on stability is performed through our search for quasi-normal modes (QNM) which are defined for purely outgoing boundary conditions. The time-domain profiles of the perturbations and the parametric variation of the QNM values are presented and discussed in some detail. Expectedly, a damped oscillatory temporal behaviour (ringdown) of the fluctuations is clearly seen through our analysis of the quasi-normal modes.
\end{abstract}

*Electronic address: surajit.basak@ifj.edu.pl, poulamiphysics@iitkgp.ac.in, sayan@phy.iitkgp.ac.in 


\section{INTRODUCTION}

The first observation of solitary waves dates back to 1834 when English naval architect John Scott Russell observed a wave in a water-canal which retained its shape while propagating over a large distance [1]. Such unique solutions to the field equations of a specific theory were later found mathematically and are known as solitary waves. If a solitary wave retains its shape even after a collision with another of its kind, then they are called solitons. Over the years, solitary waves and solitons have arisen in diverse branches of physics. Examples include optics [2, 3], cosmic strings [4, 5], monopoles [6] superconductivity [7 9], dark matter [10], fluid mechanics [11, 12], antiferromagnetism [13], particle physics [14 16] to name a few (see the cited review articles and references therein for further details on applications of solitons). Interestingly, a linear wave equation in any dimension can support non-dissipative as well as non-dispersive solutions which imply that the solutions retain their shape even if they travel from $x, t \rightarrow-\infty$ to $x, t \rightarrow \infty$. Such behaviour is evident from the dispersion relation where the angular frequency $\omega$ is a linear function of the wave number $k$. A simple scenario like this has a profound implication as it leads to the wave having equal phase and group velocity. Thus, all components of a wave packet corresponding to different frequencies travel with the same velocity keeping the shape of the wave packet intact, thereby producing a solitary wave. The situation changes drastically when an extra potential term (representing an interaction) is added to the free wave equation. Depending on the nature of this extra term, the solution of the system may loose or retain its non-dissipative or non-dispersive property. Details on these aspects which are directly linked to the ideas of solitary waves and solitons are nicely reviewed in [2, 16].

For linear equations, if one assumes no dissipation, then to retain the shape of wave packets it is mandatory for the system to be non-dispersive as well. On the other hand, for non-linear systems with no dissipation, to keep the shape of the wave packets intact, the medium has to be dispersive. Hence a dispersive medium is essential to balance the non-linearity and thus support a solitary wave.

Finding theories with non-linear equations of motion which support soliton/solitary wave solutions is easier said than done. There are some well known theories (e.g. $\phi^{4}-\phi^{2}$, sineGordon, Korteweg-de Vries, Burgers .... etc.) [2, 16 25] which do have such solutions. In general it can be said that any theory with a potential having multiple minima can support 
a solitary wave/soliton, interpolating between two neighbouring minima. Solutions represented by a monotonically increasing function are sometimes called kinks while those with a monotonically decreasing function are termed as anti-kinks. After finding such solutions the next task is to check if they have 'particle-like' features. This is done by identifying a localised energy density, a finite total energy and a verification of its stability under perturbations.

The interaction mechanisms between kink and antikink solutions vary with the solitary wave model. For instance, completely integrable solitary wave models (sine-Gordon model) have a simple interaction picture of kink-antikink collisions wherein they pass with just a phase shift or time delay. The picture is a lot more interesting in models like $\phi^{4}$ theory (nonintegrable models) where we get non-trivial interactions. The end state of collision can lead to annihilation (trapped/bound state) for low initial velocities or reflection corresponding to high velocities. Moreover, there are alternate "windows" of initial velocity called resonance windows that correspond to annihilation or reflection and have been well studied in the literature [26 31]. This interesting behaviour is called 'resonance structure' which has been observed to be suppressed by the quasi-normal modes of the system as well [32, 33].

In the following sections we will discuss a scalar field theory with a novel potential, constructed in a way to support a solitary wave solution. Our work is organised as follows. In Sec.II we construct our scalar field model with a tailor-made potential and show that it does support solitary waves. The potential has the desired double minima structure, the energy density is found to be localised and the total energy is finite and positive. The stability of the solitary waves is discussed in Sec.III. A confined harmonic oscillator potential arises when we perturb the solitary wave. Thus there are normal modes or bound states. We also explore the possible existence of transmission resonances and quasi-normal modes in the system for purely 'outgoing' boundary conditions. We end with a brief conclusion in Sec. IV.

\section{A NEW SCALAR FIELD MODEL WITH SOLITARY WAVES}

Consider a scalar field theory in (1+1)- dimensions with a Lagrangian density given as

$$
\mathcal{L}=\partial_{\mu} \phi \partial^{\mu} \phi-\tilde{V}(\phi)
$$


where $\phi$ is the scalar field having dependence on $t$ and $x$ and the potential $\tilde{V}$ is a function of $\phi$. The corresponding equation of motion, with $c=1$, is

$$
\frac{\partial^{2} \phi(x, t)}{\partial t^{2}}-\frac{\partial^{2} \phi(x, t)}{\partial x^{2}}=-\frac{d \tilde{V}}{d \phi}
$$

with the total energy

$$
E=\int_{-\infty}^{\infty} d x\left[\frac{1}{2}\left(\frac{\partial \phi}{\partial t}\right)^{2}+\frac{1}{2}\left(\frac{\partial \phi}{\partial x}\right)^{2}+\tilde{V}(\phi)\right]
$$

For the total energy to be finite, or the energy density to be localised (by localised we mean it goes to zero as $x \rightarrow \pm \infty$ ), the integrand in (3) must go to zero as $x \rightarrow \pm \infty$. Ensuring the localization of energy density would be the first step in verifying the solitary wave nature of the solution. To achieve this, the derivatives of $\phi$ and the potential $\tilde{V}(\phi)$ must reach one of its zeroes as $x \rightarrow \pm \infty$. If the potential has a minimum corresponding to $\phi=0$ only, we have a trivial solution. Hence, for non-trivial solutions with localized energy density, the potential must have atleast two minima. In the next subsection we will specifically construct such a potential satisfying the required criteria.

We mention that in this article, we have chosen to work in natural units $(h=c=1)$, which makes $\phi$ dimensionless. The potential $\tilde{V}(\phi)$ is still in units of inverse length squared. We can change our dimensionful variables $(x, t)$ to the dimensionless pair $x^{\prime}=\sqrt{V_{0}} x$ and $t^{\prime}=\sqrt{V_{0}} t$ where $V_{0}$ has dimensions of inverse length squared and may be associated with an overall scale for the potential. This will be clearer when we formulate the potential explicitly in Sec. II(A) (see eq.(12) ). In such dimensionless space and time variables we need not worry about units.

Rewriting the total energy in terms of $\left(x^{\prime}, t^{\prime}\right)$ we have

$$
E=\int_{-\infty}^{\infty} d x^{\prime}\left[\frac{1}{2}\left(\frac{\partial \phi}{\partial t^{\prime}}\right)^{2}+\frac{1}{2}\left(\frac{\partial \phi}{\partial x^{\prime}}\right)^{2}+V(\phi)\right]
$$

where $\tilde{V}(\phi)=V_{0} V(\phi)$ making $V(\phi)$ a dimensionless quantity. Proceeding with our aim of obtaining static solutions first, we note that the field equation reduces to

$$
\frac{d^{2} \phi\left(x^{\prime}\right)}{d x^{\prime 2}}=\frac{d V}{d \phi}
$$

Since the theory is relativistically invariant, once we find a localised solution of eq.(5), we can boost it to a frame where the same solution will appear to be propagating with an unchanged profile, thereby representing a solitary wave. Multiplying the earlier equation on 
both sides by $\frac{d \phi}{d x^{\prime}}$, integrating, and remembering that $\frac{d \phi}{d x^{\prime}}$ goes to one of the minima of $V(\phi)$ at $x^{\prime} \rightarrow \pm \infty$, one arrives at a first order differential equation,

$$
\frac{d \phi}{d x^{\prime}}= \pm \sqrt{2 V(\phi)}
$$

The solution to the equation (6) can be identified as $\phi_{k}\left(x^{\prime}\right)$. The R.H.S can either be always positive or always negative. Thus, for any solitary wave, the static solution is always monotonically increasing between two successive minima of the potential. The monotonically decreasing solution is called an anti-soliton.

\section{A. Constructing the potential $V(\phi)$}

In order to obtain solitary wave solutions in a scalar field theory, we need a suitable form of the potential $\mathrm{V}(\phi)$. The novelty in our work is the use of a potential which is different from the known ones yielding solitary waves. We follow a specific route in obtaining the potentialthe reason behind this way of arriving at $V(\phi)$ will become clear later. Differentiating the static equation (15) with respect to $x^{\prime}$ we get,

$$
\frac{d^{3} \phi_{k}}{d x^{\prime 3}}=\frac{d^{2} V\left(\phi_{k}\right)}{d \phi_{k}^{2}} \frac{d \phi_{k}}{d x^{\prime}}=U\left(x^{\prime}\right) \frac{d \phi_{k}}{d x^{\prime}}
$$

where all the $\phi^{\prime} s$ have been replaced by the static solution $\phi_{k}$ since we wish to have a $V(\phi)$ which will solve eq.([6) with $\phi_{k}\left(x^{\prime}\right)$. We demand $U\left(x^{\prime}\right)$ to be in the form of harmonic potential-this being the central idea behind our construction of $V(\phi)$. Replacing $U\left(x^{\prime}\right)$ by $\alpha\left(\alpha x^{\prime 2}-1\right)$ and assuming $\frac{d \phi_{k}}{d x^{\prime}}=z$ we obtain,

$$
\frac{d^{2} z}{d x^{\prime 2}}=\alpha\left(\alpha x^{\prime 2}-1\right) z
$$

The reason for such a specific demand and the role of $U\left(x^{\prime}\right)$ will be evident in section III (see eq.(21) ) where we discuss fluctuations. In short, the solvability of the fluctuation equation is behind this choice. Similar attempts have been discussed earlier in [32], [33].

A solution to the above equation (18) is $z=e^{-\frac{\alpha x^{2}}{2}}$. By integrating further, we arrive at an expression for $\phi_{k}$ as

$$
\phi_{k}=\int e^{-\frac{\alpha x^{\prime 2}}{2}} d x^{\prime}=\sqrt{\frac{\pi}{2 \alpha}} \operatorname{erf}\left(\sqrt{\frac{\alpha}{2}} x^{\prime}\right)
$$


where $\operatorname{erf}\left(\sqrt{\frac{\alpha}{2}} x^{\prime}\right)$ is the error function. The above equation can be inverted to get an expression for $x^{\prime}$ in terms of $\phi_{k}$, which, when compared with eq.([6) gives

$$
\int \frac{d \phi_{k}}{\sqrt{2 V\left(\phi_{k}\right)}}=x^{\prime}=\sqrt{\frac{2}{\alpha}} \operatorname{erf}^{-1}\left(\sqrt{\frac{2 \alpha}{\pi}} \phi_{k}\right)
$$

with $\operatorname{erf}^{-1}\left(\sqrt{\frac{2 \alpha}{\pi}} \phi_{k}\right)$ being the inverse error function. Differentiating the above equation with respect to $\phi_{k}$ we can get the expression for $V\left(\phi_{k}\right)$

$$
V\left(\phi_{k}\right)=\frac{1}{2} \operatorname{Exp}\left[-2\left[\operatorname{erf}^{-1}\left(\sqrt{\frac{2 \alpha}{\pi}} \phi_{k}\right)\right]^{2}\right] \text {. }
$$

We can now remove the subscript ' $k$ ' from $\phi$ and have a general definition of the potential $V(\phi)$. However, such a function does not have any minimum and is not defined on the entire real line. Hence it is not useful for constructing solitons or solitary waves. As a remedy, we have joined two quadratic functions at $\phi= \pm b$ which have well-defined minima at $\phi=a$ and $\phi=-a(a>b)$. We use the expression for $V\left(\phi_{k}\right)$ to motivate the final form of the potential $V(\phi)$, which is given below. A plot of the potential is shown in Fig.(1).

$$
V(\phi)= \begin{cases}\frac{1}{2}(\phi+a)^{2} & ;-\infty<\phi \leq-b \\ \frac{1}{2} \operatorname{Exp}\left[-2\left[\operatorname{erf}^{-1}(p \phi)\right]^{2}\right] & ;-b \leq \phi \leq b \\ \frac{1}{2}(\phi-a)^{2} & ; b \leq \phi<\infty\end{cases}
$$

with $\left[e r f^{-1}(p \phi)\right]^{2}$ being the square of the inverse error function and $p$ an independent parameter introduced while generalizing from $V\left(\phi_{k}\right)$ to $V(\phi)$. If one wishes to revert back to dimensional variables, $V(\phi)$ will be scaled up by factor of $V_{0}$, which has units of inverse length squared.

The potential is constructed in such a way that $V(\phi)=V(-\phi)$. While choosing the parameters, the continuity of both $\mathrm{V}$ and its derivative at $\phi= \pm b$ must be ensured. Thus, we are lead to the following conditions:

$$
\begin{gathered}
(b-a)^{2}=\operatorname{Exp}\left[-2\left[e r f^{-1}(p b)\right]^{2}\right] \\
b-a=-\sqrt{\pi} p \operatorname{Exp}\left[-\left[e r f^{-1}(p b)\right]^{2}\right] \operatorname{erf} f^{-1}(p b) .
\end{gathered}
$$

Eq.(13) corresponds to the continuity of $V(\phi)$ at $\phi= \pm b$. While taking the square root in eq.(13) we must remember that $a>b$. For eq.(14), the odd nature of the inverse error function leads to the same equation for $\phi= \pm b$. Among the three independent parameters 


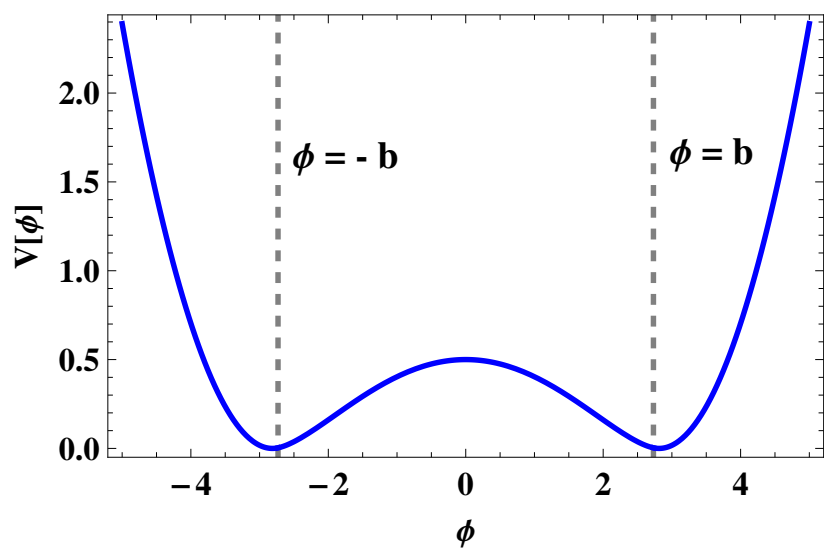

FIG. 1: The potential having two minima at $\phi= \pm a$. The values of the parameters used in the plot are mentioned in eq.(15).

namely $a, b, p$, two can be fixed by the above equations while the other needs to be fixed by hand. An additional scaling parameter $V_{0}$ (having inverse length squared units) will appear in the expression for the potential, if one is working with dimensionful variables. It may seem that the introduction of the parameter $p$ is superfluous. However, without $p$ (i.e. $p=1$ ), eqns. (13) and (14) completely fix $a$ and $b$ and the generality of the model is lost, which is not desirable. Another fact to be noted is that our potential $\mathrm{V}(\phi)$ has different functional dependencies on the field $\phi$ in different regions. Hence it will transform differently under rescaling of $\phi$ in different regions. Such a feature is not really forbidden and can only be tested if, in any way, our model becomes useful in a real and experimentally realisable context. In addition, this feature of $V(\phi)$ does explain the significance of the parameter $p$ introduced above. In the forthcoming discussions, we will use the following values for the parameters wherever necessary, if not specified otherwise.

$$
a=2.81354, b=2.73146, p=0.356825 \text {. }
$$

There is no specific advantage with the choice of these values and other sets of values for the parameters can always be chosen. With the chosen values of all parameters, the equality of eq.(13) and eq.(14) is respected upto 6 decimal places. 


\section{B. The solitary wave solution}

With the specific form of the potential in hand, we can now proceed to solving the static equation for $\phi$ (eq.([6) ) with the form of the potential $V(\phi)$ given in eq.(12). The stationary solution for the specific ranges of $x^{\prime}$ turns out to be of the following form

$$
\phi= \begin{cases}-a-(b-a) \operatorname{Exp}\left[\left(x^{\prime}+L^{\prime}\right)\right] & ;-\infty<x^{\prime} \leq-L^{\prime} \\ \frac{1}{p} \operatorname{erf}\left(\frac{\sqrt{\pi}}{2} p x^{\prime}\right) & ;-L^{\prime} \leq x^{\prime} \leq L^{\prime} \\ a+(b-a) \operatorname{Exp}\left[-\left(x^{\prime}-L^{\prime}\right)\right] & ; L^{\prime} \leq x^{\prime}<\infty\end{cases}
$$

where $x^{\prime}=L^{\prime}$ corresponds to the point $\phi=b$, when the potential $V$ is expressed in terms

of $x^{\prime} . x^{\prime}=L^{\prime}$ corresponds to $x=L$ with $L^{\prime}=\sqrt{V_{0}} L$. While constructing the stationary solution we need to keep in mind that our solitary wave must reach $\phi \rightarrow \pm a$ i.e. the points of potential minima, asymptotically. Therefore, the $( \pm)$ sign from the square root in eq.([6) has to be chosen such that $d \phi / d x^{\prime}$ is positive everywhere. In the range $L^{\prime} \leq x^{\prime}<\infty$ we take the negative sign because the square root of the potential turns out to be $(\phi-a)$. But the values of $\phi$ begin from $\phi=b<a$. Thus, to keep the R.H.S. positive we need to take the negative sign of the square root. Now one might wonder about the case when $a \leq \phi<\infty$, where the quantity $(\phi-a)$ becomes positive again. This does not pose a problem because, though $\phi$ can go to $\infty$ as the variable in the potential, for the solitary wave, we require $\phi= \pm a$ at the infinities of $x^{\prime}$. Therefore, $(\phi-a)$ will always be less than or equal to 0 and hence the negative sign will keep $d \phi / d x^{\prime}$ positive. A similar argument will hold for the range $-\infty<x^{\prime} \leq-L^{\prime}$ where the positive sign of the square root in eq.([6) is suitable.

The value of $L^{\prime}$ can be obtained by using the fact that $\phi=b$ when $x^{\prime}=L^{\prime}$. The form of $\phi_{k}$ in the range $-L^{\prime} \leq x^{\prime} \leq L^{\prime}$ resembles the expression we had obtained earlier, with some additional factors arising due to a generalization from $V\left(\phi_{k}\right)$ to $V(\phi)$. The value of $L^{\prime}$ for our choice of parameter values turns out to be,

$$
b=\frac{1}{p} \operatorname{erf}\left(\frac{\sqrt{\pi}}{2} p L^{\prime}\right) \quad \Rightarrow L^{\prime}=5 .
$$

The form of the static solution as shown in Fig.(2a) confirms that it is indeed a solitary wave, interpolating between two neighbouring minima $(\phi= \pm a)$ and monotonically increasing in between. The form of the anti-soliton can similarly be found from eq.([6]) using the negative sign. 


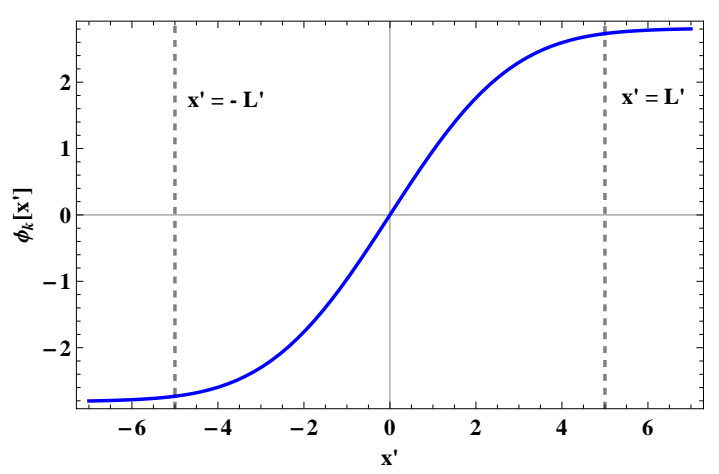

(a) Static solution starts from one minima of the potential $(\phi=-a)$ at $x^{\prime} \rightarrow-\infty$

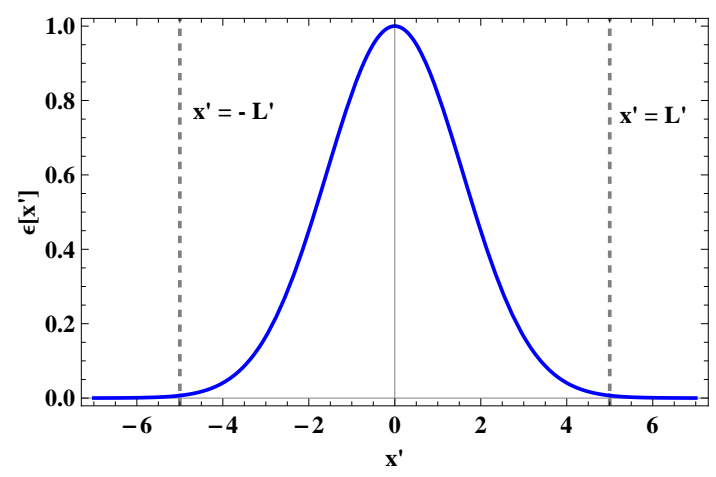

(b) Energy density centred at $x^{\prime}=0$ and dying off on both sides. and ends at the other $(\phi=a)$ at $x^{\prime} \rightarrow \infty$.

FIG. 2: In figure (a) and (b) the dashed line denotes the point $x^{\prime}=L^{\prime}$. At $x^{\prime}=L^{\prime}$ in fig.(a) the value of $\phi$ is $b$. In all the figures, previously assumed values of the parameters (eq.(15)) has been used.

The energy density for the solitary waves can be obtained using eq.(44) and eq.(6) which gives us the function, $2 V\left(\phi_{k}\right)$. The expression for energy density $\epsilon\left(x^{\prime}\right)$ as obtained for different ranges of $x^{\prime}$, by substituting $\phi_{k}$ from eq.(16) in $V\left(\phi_{k}\right)$, is centred at $x^{\prime}=0$ and decays on either side. It is given as,

$$
\epsilon\left(x^{\prime}\right)= \begin{cases}(b-a)^{2} \operatorname{Exp}\left[2\left(x^{\prime}+L^{\prime}\right)\right] & ;-\infty<x^{\prime} \leq-L^{\prime} \\ \operatorname{Exp}\left[-\frac{\pi}{2} p^{2} x^{\prime 2}\right] & ;-L^{\prime} \leq x^{\prime} \leq L^{\prime} \\ (b-a)^{2} \operatorname{Exp}\left[-2\left(x^{\prime}-L^{\prime}\right)\right] & ; L^{\prime} \leq x^{\prime}<\infty .\end{cases}
$$

Fig.(2b) demonstrates the localized nature of the energy density of the solitary wave. Integrating, we find the total energy to be,

$$
\begin{aligned}
E & =\int_{-\infty}^{\infty} 2 V\left(\phi_{k}\right) d x^{\prime} \\
& =2(b-a)^{2} \int_{L^{\prime}}^{\infty} \operatorname{Exp}\left[-2\left(x^{\prime}-L^{\prime}\right)\right] d x^{\prime}+\int_{-L^{\prime}}^{L^{\prime}} \operatorname{Exp}\left[\frac{-\pi p^{2} x^{\prime 2}}{2}\right] d x^{\prime} \\
& =\frac{\sqrt{2}}{p} \operatorname{erf}\left(\sqrt{\frac{\pi}{2}} L^{\prime} p\right)+(b-a)^{2}=3.96386 .
\end{aligned}
$$

Note that the value quoted above (for the chosen values of the parameters as mentioned in eq.(15)) is indeed positive and finite. The energy density and total energy will be scaled up 
with $V_{0}$ and $\sqrt{V_{0}}$, respectively if we work with $(x, t)$ variables. In that case, energy will be in inverse length units.

The above discussion shows that with our chosen potential we do end up with solitary waves having localised energy density and finite total energy. In addition, by construction, we are assured of the stability of the solution, as we now explain in detail in the following section.

\section{STABILITY OF SOLITARY WAVES}

As is well known, solitary waves exhibit particle-like behaviour, a feature manifest in the nature of the solution. We would now like to investigate the stability of the solitary waves obtained in the previous section, under small time-dependent perturbations. In other words, we add a small perturbation $\eta\left(x^{\prime}\right) e^{i \omega^{\prime} t^{\prime}}$ to the static solution, i.e.

$$
\phi\left(x^{\prime}, t^{\prime}\right)=\phi_{k}\left(x^{\prime}\right)+e^{i \omega^{\prime} t^{\prime}} \eta\left(x^{\prime}\right)
$$

where $\omega^{\prime}$ is a dimensionless frequency related to the dimensionful frequency via $\omega^{\prime 2}=\frac{\omega^{2}}{V_{0}}$ with $V_{0}$ in units of inverse length squared. The total field $\phi\left(x^{\prime}, t^{\prime}\right)$ still has to obey the time dependent equation (2), scaled appropriately. Substituting (20) in eq.(2), expanding $V(\phi)$ around the static solution $\phi=\phi_{k}$ and keeping terms only upto first order in $\eta$, we find,

$$
\left[-\frac{d^{2}}{d x^{\prime 2}}+U\left(x^{\prime}\right)\right] \eta\left(x^{\prime}\right)=\omega^{\prime 2} \eta\left(x^{\prime}\right)
$$

where $U\left(x^{\prime}\right)=\left.\frac{d^{2} V}{d \phi^{2}}\right|_{\phi=\phi_{k}}$. For the solitary wave to be stable under perturbation, the

Schrödinger-like equation (21) must admit solutions with positive and real values of $\omega^{\prime 2}$ (normal modes).

\section{A. Confined harmonic oscillator as effective potential}

From the way in which we constructed $V(\phi)$ in Sec.II $(\mathrm{A})$, we already have $U\left(x^{\prime}\right)$ in the following form

$$
\left.\frac{d^{2} V}{d \phi^{2}}\right|_{\phi=\phi_{k}}=U\left(x^{\prime}\right)= \begin{cases}1 & ;-\infty<x^{\prime}<-L^{\prime} \\ \alpha\left(\alpha x^{2}-1\right) & ;-L^{\prime} \leq x^{\prime} \leq L^{\prime} \\ 1 & ; L^{\prime}<x^{\prime}<\infty\end{cases}
$$


where $\alpha=\frac{\pi}{2} p^{2}$ and $L^{\prime}$ can be written as a function of $\alpha$ as $L^{\prime}=1 / \alpha$. The parameter values chosen satisfy the conditions required for a solitary wave. The plot in Fig.(3) shows the harmonic oscillator potential bounded by constant potential walls on both sides. It is interesting to note that even though $V(\phi)$ is continuous at the points $x^{\prime}= \pm L^{\prime}, U\left(x^{\prime}\right)$ is not. This is because of the fact that the quantity $\alpha$ is pre-determined from our requirement of a continuous $V(\phi)$. It can be checked that there are no values of parameters $p, a, b$ that can simultaneously make both $V(\phi)$ and $U\left(x^{\prime}\right)$ continuous. The nature of the potential $U\left(x^{\prime}\right)$ is reminiscent of a square well, except that the depth of the well now varies within the region of confinement.

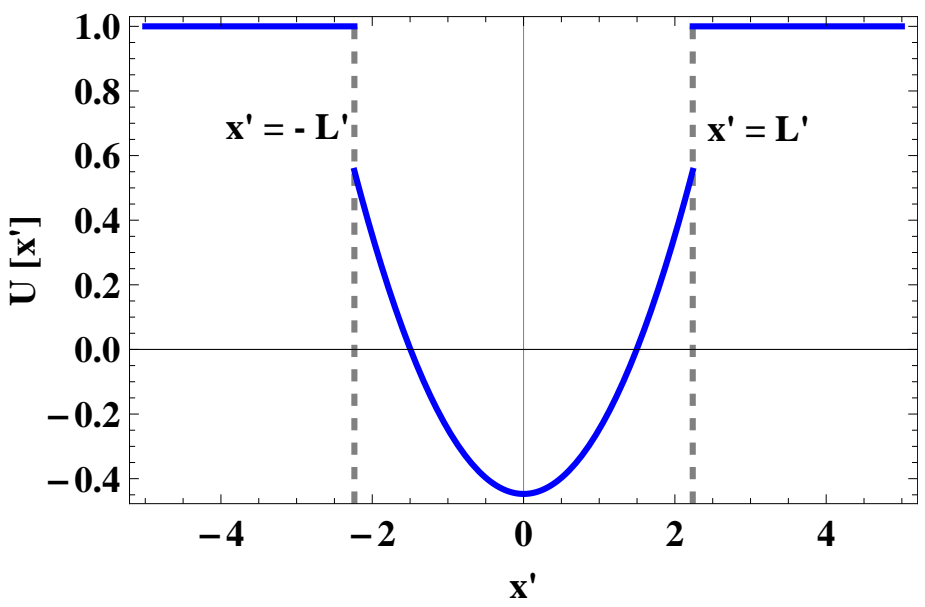

FIG. 3: The harmonic oscillator potential confined between two walls of finite height. Here $\alpha=0.2$ and $L^{\prime}=5$ following the values mentioned in eq.(15).

The general solution for $\eta\left(x^{\prime}\right)$, that dies off as $x^{\prime} \rightarrow \pm \infty$ is

$$
\eta\left(x^{\prime}\right)= \begin{cases}A e^{-m x^{\prime}} & ; L^{\prime} \leq x^{\prime}<\infty \\ B f\left(x^{\prime}\right)+C g\left(x^{\prime}\right) & ;-L^{\prime} \leq x^{\prime} \leq L^{\prime} \\ D e^{m x^{\prime}} & ;-\infty<x^{\prime} \leq-L^{\prime}\end{cases}
$$

where $m=\sqrt{1-\omega^{\prime 2}}$ and $f\left(x^{\prime}\right), g\left(x^{\prime}\right)$ are the solutions of the equation

$$
\left[-\frac{d^{2}}{d x^{\prime 2}}+\alpha\left(\alpha x^{\prime 2}-1\right)\right] \eta\left(x^{\prime}\right)=\omega^{\prime 2} \eta\left(x^{\prime}\right)
$$

and are found to be,

$$
\begin{array}{r}
f\left(x^{\prime}\right)=e^{\frac{\alpha x^{\prime 2}}{2}}{ }_{1} F_{1}\left(\frac{1}{2}+\frac{\omega^{\prime 2}}{4 \alpha} ; \frac{1}{2} ;-\alpha x^{\prime 2}\right) \\
g\left(x^{\prime}\right)=x^{\prime} e^{\frac{\alpha x^{\prime 2}}{2}}{ }_{1} F_{1}\left(1+\frac{\omega^{\prime 2}}{4 \alpha} ; \frac{3}{2} ;-\alpha x^{\prime 2}\right)
\end{array}
$$


with ${ }_{1} F_{1}$ being the confluent hypergeometric function. Note that for the first solution $f\left(x^{\prime}\right)$ we have $f\left(x^{\prime}\right)=f\left(-x^{\prime}\right)$ and $f^{\prime}\left(x^{\prime}\right)=-f^{\prime}\left(-x^{\prime}\right)$ while the reverse is true for the second solution, i.e. $g\left(x^{\prime}\right)=-g\left(-x^{\prime}\right)$ and $g^{\prime}\left(x^{\prime}\right)=g^{\prime}\left(-x^{\prime}\right)$.

The continuity of $\eta\left(x^{\prime}\right)$ and its derivative at $x^{\prime}= \pm L^{\prime}$ along with the use of the above data, results in the following equations,

$$
\begin{aligned}
& B f\left(L^{\prime}\right)+C g\left(L^{\prime}\right)=A e^{-m L^{\prime}} \quad B f^{\prime}\left(L^{\prime}\right)+C g^{\prime}\left(L^{\prime}\right)=-m A e^{-m L^{\prime}} \\
& B f\left(L^{\prime}\right)-C g\left(L^{\prime}\right)=D e^{-m L^{\prime}} \quad-B f^{\prime}\left(L^{\prime}\right)+C g^{\prime}\left(L^{\prime}\right)=m D e^{-m L^{\prime}} .
\end{aligned}
$$

We have to find values of $\omega^{\prime 2}$ for which the equations in (26) are satisfied. After some straightforward algebra, we arrive at

$$
\begin{array}{ll}
2 B f\left(L^{\prime}\right)=(A+D) e^{-m L^{\prime}} & 2 B f^{\prime}\left(L^{\prime}\right)=-m(A+D) e^{-m L^{\prime}} \\
2 C g\left(L^{\prime}\right)=(A-D) e^{-m L^{\prime}} & 2 C g^{\prime}\left(L^{\prime}\right)=-m(A-D) e^{-m L^{\prime}} .
\end{array}
$$

From the first set of equations in (27) it is evident that if $B \neq 0$ and $A \neq-D$, we have

$$
\frac{f^{\prime}\left(L^{\prime}\right)}{f\left(L^{\prime}\right)}=-m .
$$

Similarly, from the second set of equations we get a condition for $g\left(L^{\prime}\right)$ if $C \neq 0$ and $A \neq D$,

$$
\frac{g^{\prime}\left(L^{\prime}\right)}{g\left(L^{\prime}\right)}=-m \text {. }
$$

Both the equations (28) and (29) cannot be solved simultaneously for any real value of $m$. Therefore, we have to deal separately with the two cases at hand. Since the above equations for $\omega^{\prime 2}$ are transcendental, they can only be solved graphically or numerically. For each equation, we first plot the LHS and RHS in the same grid using Mathematica and find the intersection points which are solutions to the corresponding equation. The exact numerical value can then be found using the FindRoot command in Mathematica 10.

Case I: $B \neq 0, A \neq-D \& C=0, A=D$

There are two solutions for $\omega^{\prime 2}$ satisfying eq. (28), one with $\omega^{\prime 2}=0(m=1)$ and the other with $\omega^{\prime 2}=0.785064(m=0.463612)$. We have used the values of the parameters as given in eq.(15). Adopting normalization (not really a necessity for our purposes here), the constant coefficients can also be calculated from eq.(26) , and are found out to be $A=D=6.11895, B=0.50227$ and $A=D=2.74436, B=-0.33936$ for $\omega^{\prime 2}=0$ and 


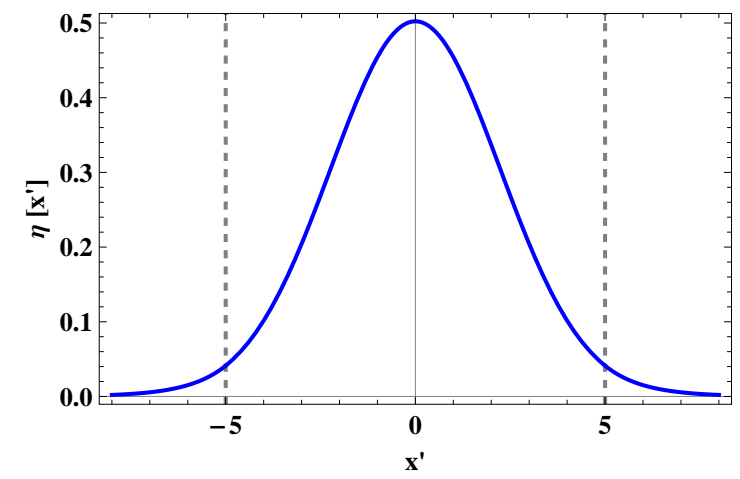

(a) The ground state $\left(\omega^{\prime 2}=0\right)$

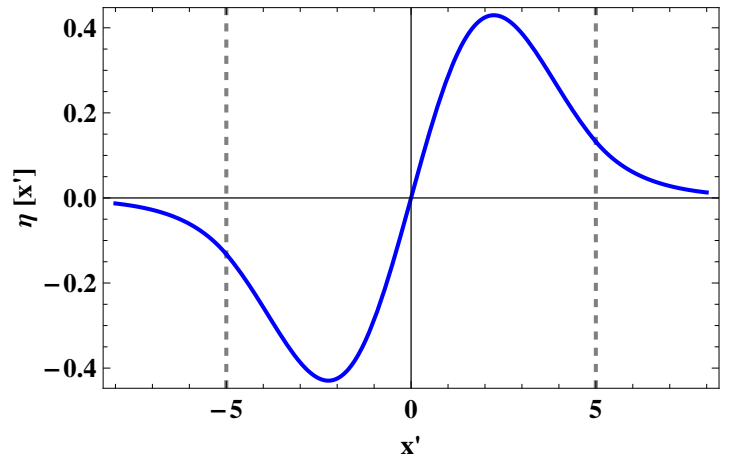

(b) First excited state $\left(\omega^{\prime 2}=0.399125\right)$

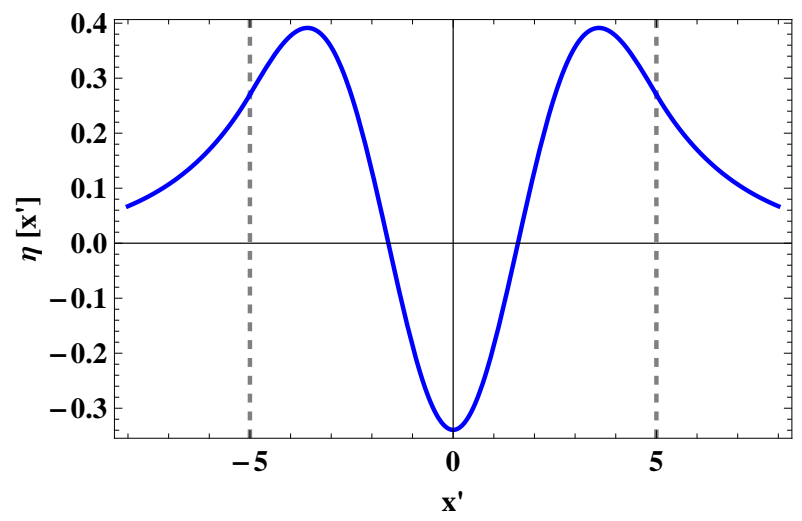

(c) Second excited $\operatorname{state}\left(\omega^{\prime 2}=0.785064\right)$

FIG. 4: Plots of wavefunctions for different energy eigenstates corresponding to $\alpha=0.2$, $L^{\prime}=5$. Dashed lines mark the position of $L^{\prime}$. Note that the increasing $\omega^{\prime 2}$ solutions have more nodes, as expected from any solution of the Schrödinger-like equation.

$\omega^{\prime 2}=0.78506$ respectively

Case II: $C \neq 0, A \neq D \& B=0, A=-D$

In this case a single solution can be found with $\omega^{\prime 2}=0.399125(m=0.77516)$ and the coefficients are $A=-D=6.37876, C=0.316409$. The plots in Fig.(4) shows the wavefunctions for different $\omega^{\prime 2}$.

From the above discussion it is apparent that there are no negative $\omega^{\prime 2}$ solutions to eq.(21). The normal modes of the system have frequency $\omega^{\prime}$. 


\section{B. Are there quasinormal modes?}

In the previous subsection, we have considered the behaviour of the solitary wave solution under perturbations and have found the associated normal modes which guarantee stability. The perturbations with positive $\omega^{\prime}$ decay asymptotically in space and oscillate harmonically in time, indicating the stability of the system. Instead of purely real energy, we can also have modes with complex $\omega^{\prime}$ - the so-called quasi-normal modes (QNMs) associated with purely outgoing boundary conditions. Such QNMs are damped oscillations in time and have divergent behaviour in the spatial part of the wave function as $x^{\prime}$ approaches the asymptotic infinities. Assuming the fact that collision of two solitary waves produces a perturbed solitary wave, which attains equilibrium by loosing energy, we can study the approach to stability of the resultant solitary wave through an analysis of such QNMs.

A similar scenario is known in gravitational wave physics where QNMs play a major role in determining the parameters of the source and the remnant produced after a merger event [34 38]. QNMs of a BH are obtained where the cause of perturbation may not necessarily be known. Since QNMs do not depend on the cause of the perturbation, it is sufficient to study them without bothering about the exact origin of their occurence. Similarly, here we study the behaviour of a solitary wave under perturbation and observe its approach to stability (in time) by finding the associated QNMs. The perturbed solitary wave may be produced as a result of a collision of two solitary waves or there could be other origins behind the fluctuations. One possible source of QNM excitation is the process of kink-antikink collision [32, 33]. Such collisions sometimes also harbour a resonance structure which have been studied for different models in [30, 31] . The conversion of a 'shape (normal) mode' into a QNM has also been analysed. In our work we have primarily focused on the possible existence of quasi-normal modes, without providing a definite prescription or mechanism on how they may be excited.

Let us now proceed further on the analysis of the perturbations of our solitary wave solution, with the aim of knowing whether the confined harmonic oscillator potential supports QNMs. From known results on the standard square well potential [39-43] for which bound states, transmission resonances and quasinormal modes do exist we can expect that in our case we will also have all three of them. Note that our perturbation potential differs from the square well in the sense that the bottom of the potential is not a constant in the domain $\left[-L^{\prime}, L^{\prime}\right]$, 
but is function of $x^{\prime}$ (here, quadratic in $x^{\prime}$ ). This is the reason why we expect results similar to the square well.

We have used the transmission coefficient of the corresponding scattering problem to find the QNMs analytically and then verified them by numerically solving for QNMs using the direct integration approach. We must keep in mind that QNMs can be found by exactly solving the Schrodinger-like differential equation for only a handful of potentials [44, 45]. Hence our confined harmonic potential is significant in its own right as the QNMs can be found exactly with the equations being solvable in terms of confluent hypergeometric functions, making the study even more worthwhile.

\section{Time domain profiles}

Following standard tools primarily used in graviatational wave physics, we recall that the time-domain profile provides a clear hint on the evolution of the perturbation as a function of time and the existence of QNMs. It also provides us with a way to check stability, a fact confirmed if the perturbation, at a particular spatial point, decays in time. In our case too, we demonstrate how $\phi\left(x^{\prime}, t^{\prime}\right)$ evolves as a function of time at a particular spatial location. Studying the perturbations of the solitary wave $\phi\left(x^{\prime}, t^{\prime}\right)$ amounts to the study of the timedependent perturbation $\eta\left(x^{\prime}, t^{\prime}\right)=\eta\left(x^{\prime}\right) e^{i \omega^{\prime} t^{\prime}}$. We begin with the scalar wave equation given as

$$
\frac{\partial^{2} \eta\left(x^{\prime}, t^{\prime}\right)}{\partial t^{\prime 2}}-\frac{\partial^{2} \eta\left(x^{\prime}, t^{\prime}\right)}{\partial x^{\prime 2}}+V_{e f f}\left(x^{\prime}\right) \eta\left(x^{\prime}, t^{\prime}\right)=0
$$

where $V_{e f f}=U\left(x^{\prime}\right)$ is the potential defined in eq.(22). This equation is converted to lightcone coordinates $d u=d t^{\prime}-d x^{\prime}$ and $d v=d t^{\prime}+d x^{\prime}$, discretized and then integrated following the procedure as given in [37] with a Gaussian profile of the form $\eta(u, 0)=e^{-(u-10)^{2} / 100}$ as the initial condition. We arrive at the time domain profiles shown in Fig.(5) as observed at the spatial position $x^{\prime}=10$ for two sets of parameter values satisfying the solitary wave condition (eq.(13) and eq.(14)).

The characteristic ringing pattern of the QNMs can be clearly observed in Fig.(5a) and (5b) for different parameter values of the potential. Thus we can conclude from the time domain profiles that our potential of eq.(22) does indeed harbour quasi-normal frequencies. The exact values of QNMs can be extracted using different techniques which we describe in detail in the forthcoming sections. 


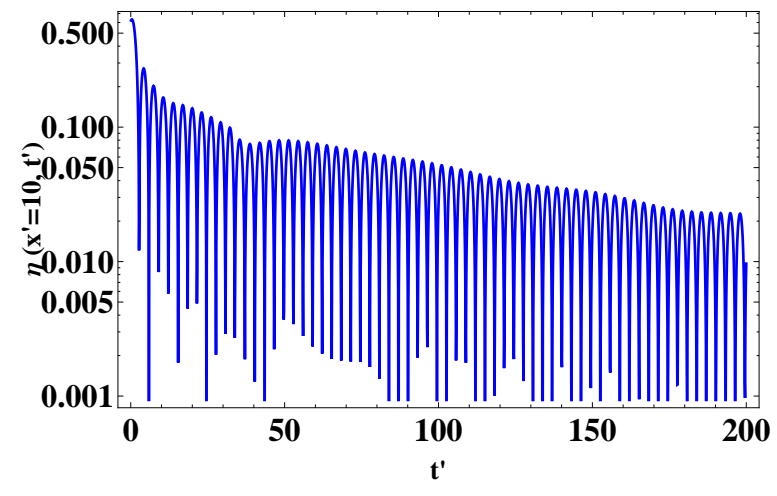

(a) $L^{\prime}=5, \alpha=0.2(p=0.356825)$

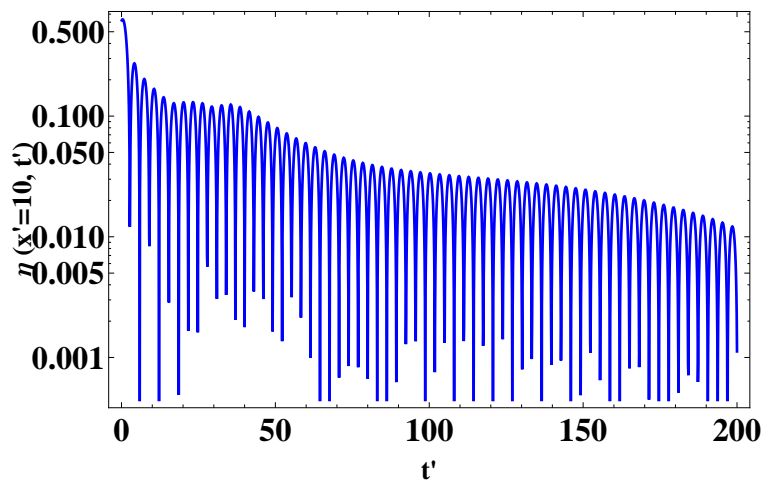

(b) $L^{\prime}=3.97887, \alpha=0.251327(p=0.4)$

FIG. 5: Time domain profiles showing the characteristic QNM ringing in semi-logarithmic scale with a Gaussian profile of the form $\eta(u, 0)=e^{-(u-10)^{2} / 100}$ as initial condition and evaluated at $x^{\prime}=10$. Both the parameter value sets satisfy the solitary wave conditions.

The field $\eta\left(x^{\prime}, t^{\prime}\right)$ decays slowly over a very long period of time. A small amount of backscattering is also observed in the time evolution plots which leads to a slight increase in amplitude of the wave. This echo-like effect can be attributed to the non-zero value of the potential (which is unity in dimensionless variables) at spatial infinity which reflects back part of the wave. Even though our potential consists of a well, we do not observe any instability for any parameter values which is also evident from the time domain profiles. Thus, our results indicate the approach to stability of our perturbed solitary wave solution. We move on now towards obtaining the quasi-normal modes.

\section{QNMs from transmission coefficient}

By definition, quasinormal modes obey purely outgoing boundary condition i.e. $\eta\left(x^{\prime}, t^{\prime}\right) \rightarrow$ $e^{-i s\left|x^{\prime}\right|} e^{i \omega^{\prime} t^{\prime}}$ with $s=\sqrt{\omega^{2}-1}$ as $x^{\prime} \rightarrow \pm \infty$. The quasinormal frequency, $\omega^{\prime}$, is expected to have its imaginary component positive, so that the temporal part decays in time $\left(\sim e^{-\operatorname{Im}\left(\omega^{\prime}\right) t^{\prime}}\right)$ corresponding to a physically stable situation while the spatial part $\operatorname{diverges}\left(\sim e^{\operatorname{Im}(s)\left|x^{\prime}\right|}\right)$ asymptotically. One way to computing such modes is to find the points where the complex transmission coefficient in the scattering problem for the Schrödinger-like equation (21) 
diverges. For such a one-dimensional scattering problem we have,

$$
\eta\left(x^{\prime}\right)= \begin{cases}A e^{i s x^{\prime}}+B e^{-i s x^{\prime}} & ;-\infty<x^{\prime} \leq-L^{\prime} \\ C f\left(x^{\prime}\right)+D g\left(x^{\prime}\right) & ;-L^{\prime} \leq x^{\prime} \leq L^{\prime} \\ F e^{-i s x^{\prime}} & ; L^{\prime} \leq x^{\prime}<\infty\end{cases}
$$

where $f\left(x^{\prime}\right)$ and $g\left(x^{\prime}\right)$ are, as defined earlier (see eq.(25) ). Note that the definitions of scattering modes and transmission coefficient are a little different from the conventional way (quantum mechanics), due to a different choice of time dependence $\left(e^{i \omega^{\prime} t^{\prime}}\right.$ instead of $\left.e^{-i \omega^{\prime} t^{\prime}}\right)$. Here, $B e^{-i s x^{\prime}}$ is the incoming wave from the left, approaching the potential. After some standard calculations, the transmission coefficient turns out to be,

$$
\tilde{t}=\frac{F}{B}=i s e^{2 i s L^{\prime}} \frac{f\left(L^{\prime}\right) g^{\prime}\left(L^{\prime}\right)-f^{\prime}\left(L^{\prime}\right) g\left(L^{\prime}\right)}{\left[i s g\left(L^{\prime}\right)+g^{\prime}\left(L^{\prime}\right)\right]\left[i s f\left(L^{\prime}\right)+f^{\prime}\left(L^{\prime}\right)\right]}
$$

The boundary conditions for QNMs demand only outgoing waves at spatial infinities, so the incident wave amplitude have to be set to zero in order to obtain the QNMs. Thus, the transmission coefficient diverges since the denominator vanishes. The equation from which we obtain the QNMs is therefore given as,

$$
-\left(\omega^{\prime 2}-1\right) f\left(L^{\prime}\right) g\left(L^{\prime}\right)+f^{\prime}\left(L^{\prime}\right) g^{\prime}\left(L^{\prime}\right)+i \sqrt{\omega^{\prime 2}-1}\left[g\left(L^{\prime}\right) f^{\prime}\left(L^{\prime}\right)+f\left(L^{\prime}\right) g^{\prime}\left(L^{\prime}\right)\right]=0 .
$$

Eqn.(33) is thus an analytical expression which contains all the information on QNMs. Only certain discrete values of $\omega^{\prime}$ will satisfy the above transcendental equation which gives the exact QNM frequencies of the system. To find the roots, we have plotted the real and imaginary parts of eq.(33) using ContourPlot in Mathematica. The intersection points are then used as initial values in FindRoot which leads to the exact values of the QNM frequencies.

Apart from the QNMs, we can also observe the transmission resonances for real $\omega^{\prime 2}$ in scattering (see Fig.(6) ), where the transmission probability $T=|\tilde{t}|^{2}$ reaches its maximum value i.e. one. For such frequencies, the potential well becomes totally transmitting with no reflection. Transmission resonances for various potentials are crucial in different contexts [46-48]. However, here we will not discuss them any further. Instead, we seek to verify the QNMs obtained using a different method, i.e. direct integration. 


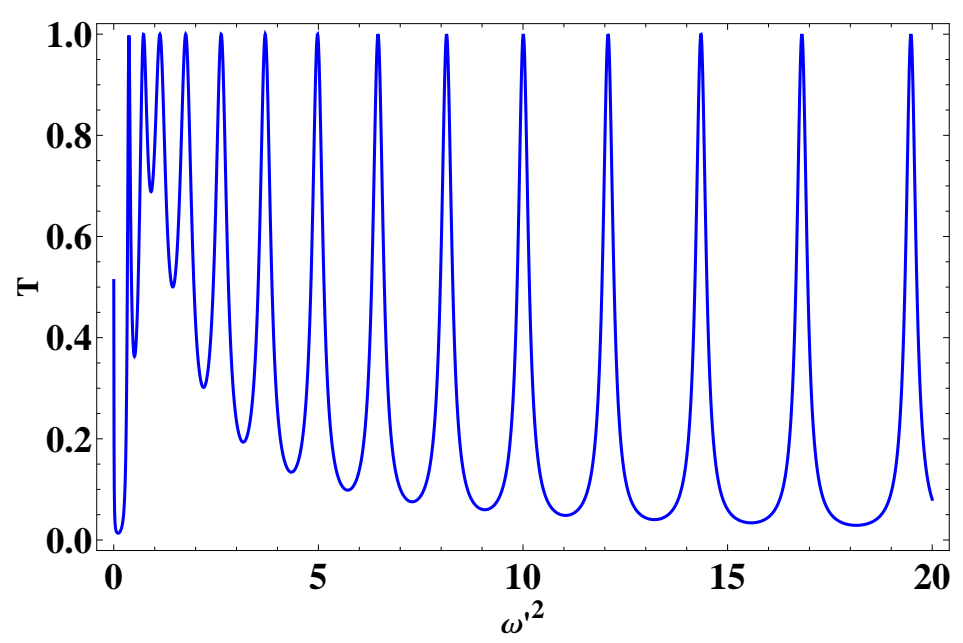

FIG. 6: Transmission resonance as observed for $L^{\prime}=5, \alpha=0.2$

\section{QNMs from direct integration}

In most physical scenarios, differential equations corresponding to the QNMs are difficult to solve analytically. Entirely numerical methods are the only way out. Numerical methods also provide a way of verifying the results obtained through analytical techniques as we will see in our case here. The direct integration method, also called the 'shooting' method was first introduced by Chandrasekhar and Detweiler in [49]. Here, the Schrödinger-like differential equation (eq.(21)) is integrated straightaway, with proper boundary conditions, thereby yielding the QNMs. The method has been modified slightly while applying it to our specific potential.

The solution at infinity (or, in this case for $x^{\prime}>L^{\prime}$, i.e. positive $x^{\prime}$ ) is exactly known in terms of exponential functions

$$
\eta\left(x^{\prime}\right)=A_{1} e^{k x^{\prime}}+A_{2} e^{-k x^{\prime}}
$$

where $k=i \sqrt{\omega^{\prime 2}-1}$. Ideally the integration should be done from $-\infty$ to $\infty$ but since our potential is symmetric about the origin, we integrate from $x^{\prime}=0$ to $\infty$ and reflect the solution at the origin to arrive at the function in the region $x^{\prime}<0$ (see [50, 51]). There will be symmetric and anti-symmetric solutions corresponding to suitable matching conditions at the origin: namely, $\eta(0)=0$ for anti-symmetric and $\eta^{\prime}(0)=0$ for symmetric solutions where $\eta^{\prime}$ denotes derivative of $\eta$ with respect to $x^{\prime}$.

The differential equation (eq.(21)) is then numerically integrated from the origin to $x^{\prime}=L^{\prime}$ 
with the initial conditions: $\eta(0)=0$ for the odd case and $\eta^{\prime}(0)=0$ for even. One has to keep in mind that the potential in the region $\left[-L^{\prime}, L^{\prime}\right]$ is that of a harmonic oscillator.

Next, we take the solution obtained after integration and equate it and its derivative with the solution obtained in eq.(34) at $x^{\prime}=L^{\prime}$. This gives us expressions for $A_{1}$ and $A_{2}$ as functions of $\omega^{\prime}$ which can be substituted back to eq.(34) to get the general solution at any $x^{\prime}>L^{\prime}$. Finally, we make $A_{1}=0$ in the solution thus obtained, so that only outgoing waves exist at infinity (as per the convention used here, $A_{1}$ is associated with the incoming wave while $A_{2}$ involves the outgoing wave). The solution of this equation will give the values of the QNM frequencies. To check the consistency of our solution, we can calculate the ratio $A_{1} / A_{2}$ and see if it is nearly equal to zero. An added advantage of this method is that we can identify the QNMs as even or odd which was not possible in the transmission coefficient method discussed previously.

\section{Comparing QNMs from the two methods}

Finally we are in a position to implement the above-stated methods and calculate the QNM frequencies for different parameter values of the potential. The following tables show QNM values for certain parameter choices as indicated there.

\begin{tabular}{||c|c||}
\hline$\omega^{\prime}$ (From transmission coefficient) & $\omega^{\prime}$ (From DI) \\
\hline \hline $0.966557+\mathrm{i} 0.0537962$ & $0.966557+\mathrm{i} 0.0537962$ \\
$1.59948+\mathrm{i} 0.321305$ & $1.59948+\mathrm{i} 0.321305$ \\
$2.22103+\mathrm{i} 0.420105$ & $2.22103+\mathrm{i} 0.420105$ \\
\hline $1.28732+\mathrm{i} 0.240357$ & $1.28732+\mathrm{i} 0.240357$ \\
$1.91022+\mathrm{i} 0.377235$ & $1.91022+\mathrm{i} 0.377235$ \\
$2.53224+\mathrm{i} 0.45485$ & $2.53224+\mathrm{i} 0.45485$ \\
\hline
\end{tabular}

TABLE I: Values of parameters are: $\alpha=0.2$ and $L^{\prime}=5$. First three QNMs are odd while remaining three are even. 


\begin{tabular}{||c|c||}
\hline$\omega^{\prime}$ (From transmission coefficient) & $\omega^{\prime}$ (From DI) \\
\hline \hline $1.16485+\mathrm{i} 0.243292$ & $1.16485+\mathrm{i} 0.243292$ \\
$1.96892+\mathrm{i} 0.464461$ & $1.96892+\mathrm{i} 0.464461$ \\
$2.75861+\mathrm{i} 0.573992$ & $2.75861+\mathrm{i} 0.573992$ \\
\hline $1.57139+\mathrm{i} 0.380764$ & $1.57139+\mathrm{i} 0.380764$ \\
$2.3641+\mathrm{i} 0.52566$ & $2.3641+\mathrm{i} 0.52566$ \\
$3.15296+\mathrm{i} 0.613966$ & $3.15296+\mathrm{i} 0.613966$ \\
\hline
\end{tabular}

TABLE II: Values of parameters are: $\alpha=0.251327$ and $L^{\prime}=3.97887$. First three QNMs are odd while remaining are even.

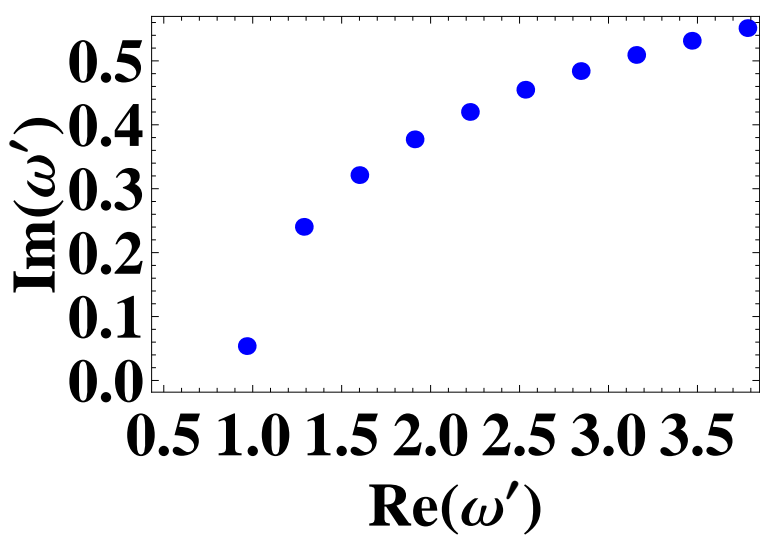

(a) $L^{\prime}=5, \alpha=0.2$

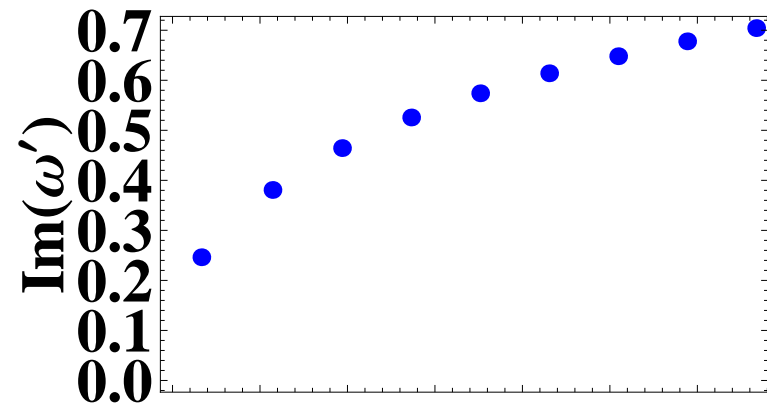

\subsection{5 2.0 2.5 3.0 3.5 4.0 $\operatorname{Re}\left(\omega^{\prime}\right)$}

(b) $L^{\prime}=3.97887, \alpha=0.251327$

FIG. 7: Variation of real and imaginary part of QNM frequencies for two sets of parameter values that satisfy the soliton criteria.

From the tables I and II, it is quite evident that the results from both the methods are consistent with each other. The parameter values chosen to obtain the results in Table I] and Table IIsatisfy solitary wave conditions mentioned earlier in eq.(13) and (14). In Fig.(17), the variation of real and imaginary parts of the QNM frequencies can be seen. All QNM frequencies have a positive imaginary part indicating the decay of perturbation with time. Hence we can conclude that the potential in eq.(22) is stable against perturbations both for 
an independent set of parameters as well as for our solitary wave solution. The results from DI match exactly, up to the decimal places considered here, with the solutions obtained using the transmission coefficient, because in DI we have not used any series expansion and all exact solutions have been incorporated. Obviously if we compare the QNM values from the two methods after the decimal places shown in the tables, we do find differences. One might also notice that even though the time domain profiles indicate a slow decay of signal, we find the imaginary parts of the QNM frequencies to be not so small as to produce such a large decay time. One possible explanation for the slow decay might be attributed to the small reflections from a non-zero $V_{0}$ or in the dimensionless variables notation, unity, at spatial infinity which induces a greater strength in the signal. Due to this additional reflected signal we do not observe a sharp damping as is usually expected in QNM frequencies. Thus it can be concluded that the time domain profile is not dominated by any individual QNM frequency of the potential. We can further verify this fact if we apply the Prony method [37] and extract the dominant frequency directly from the time evolution. This can be considered as an example of the observation made by Nollert [52] for discontinuous potentials, where it is said that even though the QNMs form a complete set, there is no single mode among the QNM frequencies that dominates time evolution.

Apart from the study of the confined harmonic oscillator potential in the context of solitary wave solution we can investigate features associated with the given $U\left(x^{\prime}\right)$, as an independent entity. A brief discussion on how the QNM frequencies depend on the parameters in $U\left(x^{\prime}\right)$ can be found in the Appendix. Obviously without the solitary wave criteria, we can freely vary the parameters $\alpha, L^{\prime}$ and see their effects. Such a study could be useful in other areas where a potential like $U\left(x^{\prime}\right)$ may arise.

\section{CONCLUSION}

Solitary waves have interested researchers from various branches of physics over many years. The property of such solutions retaining their shape while traveling, renders them as coherent structures created due to the presence of nonlinearity in the equations of motion. Finding a model non-linear theory with dispersion and no dissipation with solitary wave solutions is not an easy task. In our work we have developed a new model with a solitary wave solution in a massless scalar field theory in $1+1$ dimensions. The functional dependence 
of the potential on the scalar field $\phi$ was not defined to begin with. We constructed the potential $V(\phi)$ such that (a) our theory supports a non-trivial solitary wave solution and (b) the equation for fluctuations about the solitary wave is analytically solvable (in our case it is just a confined simple harmonic oscillator). We also checked the localisation of energy density and the finiteness of total energy for our solitary waves. In our presentation, we have used dimensionless variables $\left(x^{\prime}, t^{\prime}\right)$ and have shown how to switch over to dimensionful ones, when needed.

After obtaining the soliton solution we moved on to studying its stability by finding the normal and quasi-normal modes of scalar perturbations. As stated before, the potential $V(\phi)$ was constructed such that the perturbation potential $U\left(x^{\prime}\right)$ is in the form of a confined harmonic oscillator. That the potential is stable against perturbation was evident from the normal modes. With the calculation of QNMs we also verified the stability in time through decaying perturbations. We have obtained the QNMs analytically through the divergence of the transmission coefficient and also numerically, through direct integration. Both methods provide QNM values that match perfectly upto the decimal places considered. Finally, an interesting observation from the QNM study is the fact that no single QNM frequency dominates the time domain profile which can be attributed to the discontinuity of the potential.

In summary, our work presents a novel scalar field model with solitary waves for which the fluctuation equation is easy to analyse quantitatively. For most known solitary waves and solitons, one ends up with a fluctuation equation with a rather complicated potential, which is tedious to solve. Here, we have a model for which the solitary wave arises through a tailor-made potential with the perturbation equation simple and easy to solve and analyse. We believe there could be many similar models wherein both the solitary waves as well as its perturbations are analytically tractable, which makes the quantitative understanding of the system relatively easier. Scenarios where double-well potentials generically arise are viable candidates where our model can be of use, possibly in a physically relevant context. Finally, newer models explaining how quasinormal modes may be excited in the context of solitary waves, need to be built, in order to provide the right context wherein the possibilities (on QNMs in particular) discussed here, in this work, will be of importance, in future. 


\section{Acknowledgements}

Surajit Basak thanks Centre for Theoretical Studies, IIT Kharagpur, India for informal visits during 2019 when this work was initiated and carried out. He also thanks his present host, P. Piekarz, Institute of Nuclear Physics, Polish Academy of Sciences, Krakow, Poland for allowing him to use his present address, in this article.

\section{Appendix A: Variation of QNMs with parameters}

In this appendix we discuss the dependence of the QNM frequencies on the parameters of the potential. Such an analysis will help us in appreciating the confined harmonic potential $U\left(x^{\prime}\right)$ as an independent, discontinuous potential with its parameters not necessarily obeying the solitary wave criteria. This independent study could be of use in scenarios where a similar potential may arise. We note that the potential $U\left(x^{\prime}\right)$ involves parameters: $\alpha$ and $L^{\prime}$, which are independent if we do not demand its solitary wave connect. Hence, we may vary them freely and see how the QNM frequencies get affected. In Fig.(8) we show how the spectrum of the QNMs obtained differ when we vary each parameter separately.

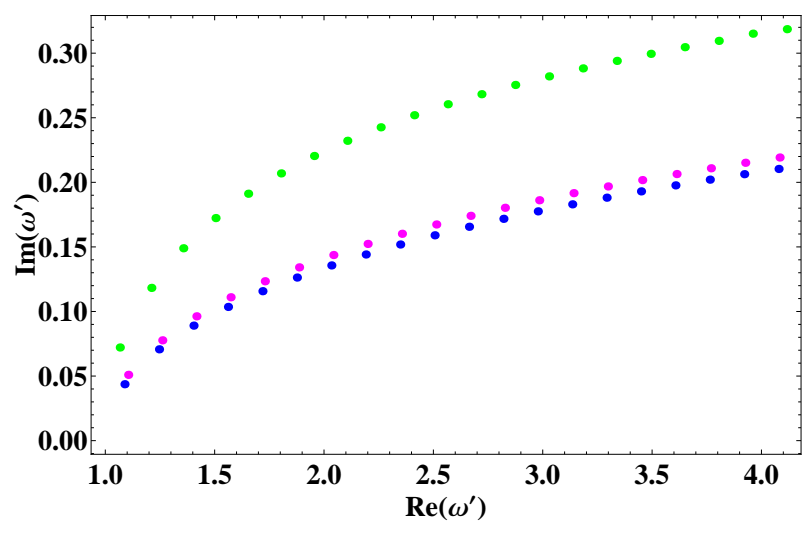

(a) $L^{\prime}=10, \alpha=0.025(\bullet), 0.05(\bullet), 0.1(\bullet)$

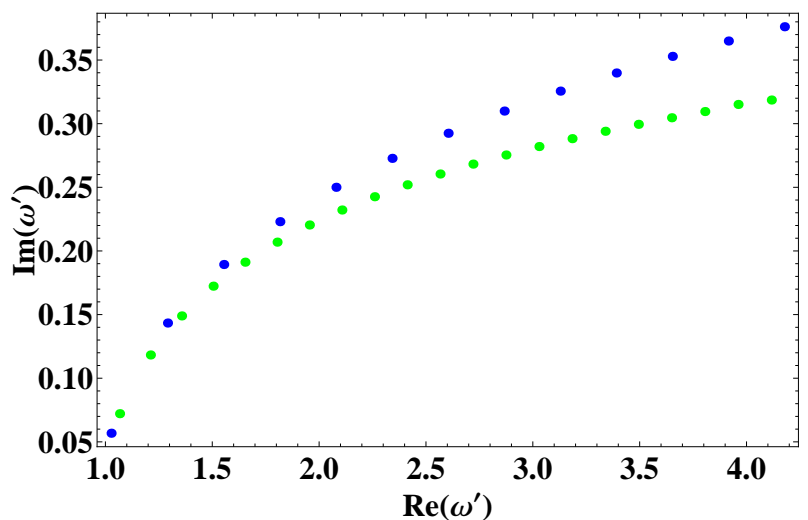

(b) $\alpha=0.1, L^{\prime}=6(\bullet), 10(\bullet)$

FIG. 8: $\operatorname{Im}\left(\omega^{\prime}\right)$ vs $\operatorname{Re}\left(\omega^{\prime}\right)$ plotted for different parameter values.

- In Fig. (8a $)$ we observe the effect of variation of $\alpha$ on the QNMs. The value of $\alpha$ determines the depth of the potential well. One must keep in mind while choosing the parameters, that we are allowed to take only those values obeying $\alpha\left(\alpha L^{\prime 2}-1\right)<1$ ( this guarantees that the nature of the confined harmonic oscillator potential is preserved). As $\alpha$ increases, the well 
becomes narrow and the imaginary part of the QNM frequency increases.

- Changing the magnitude of discontinuity or jump at $x^{\prime}= \pm L^{\prime}$ also affects the QNM spectrum as observed in Fig. (8a). If we go to lower values of $\alpha$, the well becomes shallower while its width $\left(2 L^{\prime}\right)$ remains fixed, which results in a larger discontinuity. Hence we see that small values of $\alpha$ correspond to lower values of $\omega_{i}^{\prime}$.

- When $L^{\prime}$ is varied i.e. the width of the potential increases (see Fig. (8b ) ), the imaginary part of QNM decreases. For very large $L^{\prime}$, the imaginary part will keep on getting smaller. Finally for $L^{\prime} \rightarrow \infty$ the well vanishes leaving a constant potential with no interesting features.

In general, we find the lower modes to remain almost unaffected by a change of the parameters. Also, the real part of QNMs remain nearly same under parameter variation, whereas, it is the imaginary part which shows the effect. Working in dimensionful variables would lead to the introduction of $V_{0}$ (in inverse length squared units) in the potential. Changing the magnitude of $V_{0}$ with the other parameters kept fixed will affect the discontinuity. Hence increasing $V_{0}$ or decreasing $\alpha$ will have the same effect on the QNM spectrum.

[1] J.E.Allen, The Early History of Solitons (Solitary Waves), Physica Scripta. Vol. 57, 436 441 ( 1998).

[2] R. Rajaraman, Solitons and Instantons, North Holland (1982).

[3] F.Abdullaev, S.Darmanyan, P.Khabibullaev, J. Engelbrecht, Optical Solitons, Springer, New York (2014), Y. Song, X. Shi, C. Wu, D. Tang, H. Zhang, Recent progress of study on optical solitons in fiber lasers, Applied Phys. Rev. 6, 021313 (2019), Z. Chen, M. Segev, D. N. Christodoulides, Optical spatial solitons: historical overview and recent advances, Rep. Prog. Phys. 75086401 (2012).

[4] J.Garriga, E.Verdaguer, Cosmic Strings and Einstein-Rosen Soliton Waves, Phys.Rev.D 36 2250 (1987).

[5] M. Hindmarsh, K. Rummukainen, D. J. Weir, New Solutions for Non-Abelian Cosmic Strings, Phys. Rev. Lett. 117, 251601 (2016).

[6] R. Hivet, H. Flayac, D. D. Solnyshkov, D. Tanese, T. Boulier, D. Andreoli, E. Giacobino, J. 
Bloch, A. Bramati, G. Malpuech, A. Amo, Half-solitons in a polariton quantum fluid behave like magnetic monopoles, Nat. Phys. vol. 8, pg. 724-728(2012).

[7] Y.Tanaka, Soliton in Two-Band Superconductor, Phys. Rev. Lett. 88, 017002 (2001).

[8] A.A. Abrikosov, Nobel lecture: Type-II superconductors and the vortex lattice, Rev. Mod. Phys.76 975 (2004).

[9] O. M. Auslaender, L. Luan, E. WJ Straver, J. E. Hoffman, N. CKoshnick, E. Zeldov, D. A. Bonn, R. Liang, W. N. Hardy, K. A. Moler, Mechanics of individual isolated vortices in a cuprate superconductor,Nat. Phys. 5 35-39 (2009).

[10] T. Asselmeyer-Maluga, J. Kró, Dark Matter as gravitational solitons in the weak field limit, arXiv:2012.05358v1 [gr-qc] (2020), L. Arturo Ureña-López, Brief Review on Scalar Field Dark Matter Models, Front. Astron. Space Sci. vol.6 (2019), E. W. Mielke, Soliton model of dark matter and natural inflation, J. Phys.: Conf. Ser. 1208012012 (2019).

[11] R.H.J. Grimshaw, Solitary waves in fluids, WIT Press (2007).

[12] M.A. Helal, Soliton solution of some nonlinear partial differential equations and its applications in fluid mechanics, Chaos, Solitons and Fractals, vol.13, issue 9 (2002).

[13] E. G. Galkina, B. A. Ivanov, Dynamic solitons in antiferromagnets (Review Article), Low Temp. Phys. 44, 618 (2018).

[14] C. Rebbi, G. Soliani, Solitons and Particles, World Scientific Publishing Co. (1984).

[15] R.A. Pakula, Solitons and Quantum Behavior, arXiv:1612.00110 [quant-ph].

[16] S. Coleman, Aspects of symmetry, Cambridge University Press (1988).

[17] D. J. Korteweg, G. de Vries, On the Change of Form of Long Waves Advancing in a Rectangular Canal, and on a New Type of Long Stationary Waves, Philosophical Magazine, 39 (240): 422-443 (1895)

[18] T. Sugiyama, Kink-antikink collisions in the two-dimensional $\phi^{4}$ model, Progress of Theoretical Physics, 61(5), 1550-1563, (1979).

[19] M. A. Lohe, Soliton structures in $P(\phi)_{2}$, Physical Review D 20, 3120 (1979).

[20] D. Bazeia, E. Belendryasova, V. A. Gani, Scattering of kinks of the sinh-deformed $\phi^{4}$ model, Eur. Phys. J. C 78340 (2018).

[21] A. R. Gomes, F. C. Simas, K. Z. Nobrega, P. P. Avelino, False vacuum decay in kink scattering, JHEP 10, 192 (2018).

[22] V. A. Gani, V. Lensky, M. A. Lizunova, Kink excitation spectra in the $(1+1)$-dimensional $\phi^{8}$ 
model, JHEP 08, 147 (2015).

[23] A. Alonso-Izquierdo, Reflection, transmutation, annihilation, and resonance in two-component kink collisions, Phys. Rev. D 97, 045016 (2018).

[24] V. A. Gani, A. M. Marjaneh, A. Askari, E. Belendryasova, D. Saadatmand, Scattering of the double sine-Gordon kinks, Eur. Phys. J. C 78345 (2018).

[25] A.R. Gomes, R. Menezes, J.C.R.E. Oliveira, Highly interactive kink solutions, Phys. Rev. D 86(2), 025008 (2012).

[26] V. A. Gani, A. E. Kudryavtsev, M. A. Lizunova, Kink interactions in the (1+ 1)-dimensional $\phi^{6}$ model, Physical Review D 89(12), 125009, (2014).

[27] E. Belendryasova, V. A. Gani, Scattering of the $\phi^{8}$ kinks with power-law asymptotics, Commun. Nonlinear Sci. Numer. Simulat. 67414 (2019).

[28] D. Bazeia, A. R. Gomes, K. Z. Nobrega, F. C Simas, Kink scattering in a hybrid model, Phys. Lett. B 793:26-32 (2019).

[29] A. Demirkaya, R. Decker, P. G. Kevrekidis, I. C. Christov, A. Saxena, Kink dynamics in a parametric $\phi^{6}$ system: a model with controllably many internal modes, JHEP 12:71 (2017).

[30] D. K.Campbell, J. F.Schonfeld, C. A. Wingate, Resonance structure in kink-antikink interactions in $\phi^{4}$ theory, Physica D: Nonlinear Phenomena, 9(1-2), 1-32 (1983).

[31] A. Halavanau, T. Romanczukiewicz, Ya. Shnir, Resonance structures in coupled twocomponent $\phi^{4}$ model, Phys. Rev. D 86, 085027 (2012).

[32] J.G.F Campos, A. Mohammadi, Quasinormal modes in kink excitations and kink-antikink interactions: a toy model,arXiv: 1905.00835vl, Eur. Phys. J. C 80,352,(2020).

[33] P. Dorey and T. Romanczukiewicz, Resonant kink-antikink scattering through quasinormal modes, Physics Letters B 779, 117 (2018).

[34] C. V. Vishveshwara, Scattering of gravitational radiation by a Schwarzschild black hole, Nature 227, 936 (1970).

[35] C. V. Vishveshwara, Stability of the Schwarzschild metric, Phys. Rev. D 1, 2870 (1970).

[36] K.D. Kokkotas, B.G. Schmidt, Quasi-Normal Modes of Stars and Black Holes, Living Rev. Relativ. 2, 2 (1999).

[37] R.A. Konoplya and A. Zhidenko, Quasinormal modes of black holes: from astrophysics to string theory, Rev. Mod. Phys. 83, 793 (2011).

[38] V. Cardoso, Quasinormal Modes and Gravitational Radiation in Black Hole Spacetimes, PhD 
Thesis, arXiv:0404093[gr-qc] (2003).

[39] D.W.L. Sprung, H. Wu, J. Martorell, Poles, bound states, and resonances illustrated by the square well potential, Am.J. of Phys. 64, 136 (1996).

[40] D. Bindel, M. Zworski, Theory and Computation of Resonances in 1-D Scattering, http://www.cs.cornell.edu/ bindel/cims/resonant1d/

[41] S. Jenks, On the pole structure of the S-matrix for a square potential well, http://www.physics.drexel.edu/ jenks/Pole Structure.pdf

[42] D.J. Griffiths, Introduction to Quantum Mechanics, Second edition, Chapter 2 (pg. 78-82).

[43] F. Schwabl, Quantum Mechanics, Fourth edition, Chapter 3 pg.(71-75, 81-86).

[44] P.D. Roy, J. Das and S. Kar,Quasi-normal modes in a symmetric triangular barrier, Eur. Phys. J. Plus 134, 571 (2019).

[45] P. Boonserm and M. Visser, Quasi-normal frequencies: key analytic results, JHEP, 1103:073 (2011).

[46] S. Kar, S.N. Minwalla, D. Mishra and D. Sahdev, Resonances in the transmission of massless scalar waves in a class of wormholes, Phys. Rev. D Vol.51, No. 4 (1994).

[47] W. Chen, D. L. Mills, Gap solitons and the nonlinear optical response of superlattices, Phys. Rev. Lett. 58, 160 (1987).

[48] K. Rapedius, H. J. Korsch, Barrier transmission for the one-dimensional nonlinear Schrödinger equation: Resonances and transmission profiles, Phys. Rev. A 77, 063610 (2008).

[49] S. Chandrasekhar and S. Detweiler, The quasi-normal modes of the Schwarzschild black hole, Proc. R. Soc. Lond. A 344,441 (1975).

[50] S. Aneesh, S. Bose and S. Kar, Gravitational waves from quasinormal modes of a class of Lorentzian wormholes, Phys. Rev. D 97, 124004 (2018).

[51] P.D. Roy, S. Aneesh and S. Kar, Revisiting a family of wormholes: geometry, matter, scalar quasinormal modes and echoes, Eur. Phys. J.C 80, 850 (2020).

[52] H.P. Nollert, About the Significance of Quasinormal Modes of Black Holes, Phys. Rev. D 53, 4397 (1996). 